\title{
Advances in HREBSD for Elastic Strain Measurement and its Application to Mechanical Testing
}

\section{J Dingley ${ }^{1}$ and G M Meaden²}

${ }^{1}$ Department of Physics, University of Bristol, Tyndall Ave. Bristol BS8 1TH, UK

${ }^{2}$ BLG Productions Ltd, Centre Gate, Colston Avenue, Bristol, BS1 4TR, UK

The use of High Angular Resolution EBSD (HREBSD) for the measurement and mapping of elastic strain and geometrically necessary dislocation (GND) densities is becoming more common, especially for the study of polycrystalline materials and for in-situ straining and heating experiments. In the method, now frequently referred to as the Wilkinson method [1], multiple regions of interest of an EBSD pattern taken from a test region, are compared to a reference pattern taken from a nominally strain free region using a cross correlation procedure. In this way the relative distortion of the test pattern is determined. From this the full strain and rotation tensors are determined directly and from the rotation gradient between adjacent points in a map the GND density can be found. The current tendency is for the mapping of strain in polycrystalline specimens multiple times with intermediate thermo/ mechanical treatment and to map them over large areas. The sensitivity of the technique is 1 part in 10000 for strain measurement and 0.006 degrees for rotational measurement; this requires a pattern shift measurement equal to 0.1 of a camera pixel for a camera with $1000 \mathrm{x}$ 1000 pixels. However, in order to secure this sensitivity care has to be taken to ensure accurate subtraction from the measured differences between reference and test patterns, those differences that are due to $a /$ the beam movements needed to collect the map and b/ the zooming of the EBSD pattern arising from changes in specimen to screen distance, when scanning an area of a steeply tilted sample. It has been shown that one way to track accurately the electron beam movement is to attach to the front of the EBSD detector a circular aperture mounted parallel to the phosphor screen of the camera. The attachment is referred to as a nose cone [2]. The position of the shadow of the aperture and its diameter provide information that can be directly related to the position of the diffraction pattern centre, PC, and sample to screen distance $Z^{*}$ for all points in a scan. The current investigation has concentrated on mapping the potential errors that occur in shift measurement with and without the use of a nose cone. We also correlated data from a series of scans of a polycrystalline sample which was removed from the microscope between scans. This was done to determine the reliability of using a reference pattern recorded in the first scan for use as a reference pattern for subsequent scans.

HREBSD maps were obtained from perfect single crystal silicon specimens over areas ranging from $100 \mu \mathrm{m}$ square to $1.5 \mathrm{~mm}$ square. To test the reliability of using a single reference pattern as the reference for a sequence of scans, a polycrystalline nickel sample was mapped over an area of 15 $\mu \mathrm{m}$, removed from the microscope, remounted and mapped again. The strain maps should be identical if $\mathrm{PC}$ and $\mathrm{Z}^{*}$ positions between the two mappings are properly correlated.

As illustrated in figure 1, it was shown that the limiting distance over which the normally used shift correction procedure could be relied on, for a specimen tilted 70 degrees from the horizontal, was $\pm 150 \mu \mathrm{m}$ from the reference pattern. The figure shows the residual $\mathrm{x}$ shift for each data point after beam shift and zoom correction have been applied. The colour range in the image, red to blue, is 
\pm 0.1 pixels and missing points are those that exceed this shift range (image width $300 \mu \mathrm{m}$, reference point at centre). The shift errors outside of this range are due to small deviations of the SEM magnification from its initial calibration value and uncertainties in the sample geometry. The result of attaching a nose cone and mapping the aperture shadow movement is shown in figure 2 . It is for the case of a scan covering $800 \mu \mathrm{m}$ x $1600 \mu \mathrm{m}$ on a GaAs sample tilted at 70 degrees with beam step size of $100 \mu \mathrm{m}$. The shadows show that the beam movements did not form a rectangular scan over the specimen surface as commanded by the input scan dimensions but were in the form of a trapezium. Without the nose cone attachment this would not have been detected. The true movement of the EBSD PC can be calculated from the shadow movement [2] and is shown in figure 3. The importance of accurate location of the PC and $\mathrm{Z}^{*}$ values is that the zoom corrections are highly dependent on both of these parameters. They are also paramount when using a reference pattern from one scan as the reference pattern for a second scan. Figures $4 \mathrm{a} \& \mathrm{~b}$ are two such scans taken of the same Ni sample. The shadow data in this case showed that the PC values for the data point used as reference pattern for each the two scans, differed respectively by $\mathrm{x}: 9.5 \mu \mathrm{m}, \mathrm{y}: 20.2 \mu \mathrm{m}$ $\& \mathrm{z}: 8.1 \mu \mathrm{m}$ but their respective positions in the map differed by $\mathrm{x}: 3 \mu \mathrm{m}, \mathrm{y}: 4 \mu \mathrm{m}$. This implied a different correction was needed for PC and zoom values than would have been the case using map position correction. In the illustrated case, as there was no additional straining between the two scans, the maps should be identical. The mean difference between corresponding map positions in, for example, the resulting $\varepsilon_{11}$ strain values was \pm 0.00038 (the maps have been cropped to permit easier identification of corresponding points).
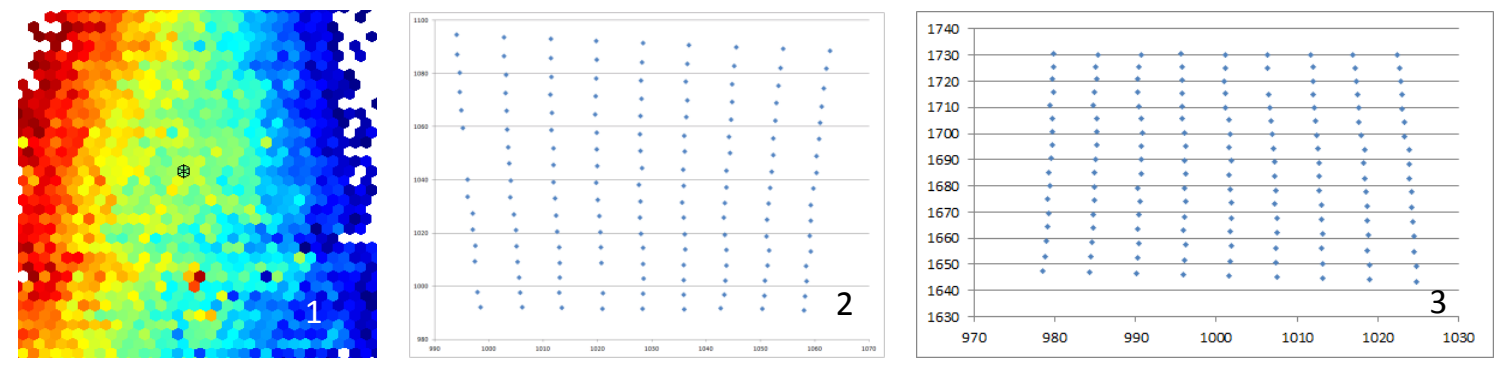

Figures 1-3, Residual $x$ shifts after subtraction of beam shifts and zoom effects, image width $300 \mu \mathrm{m}(2)$ aperutre shadow centre movemenets, (3)back calculted PC positons from measured aperture shadow
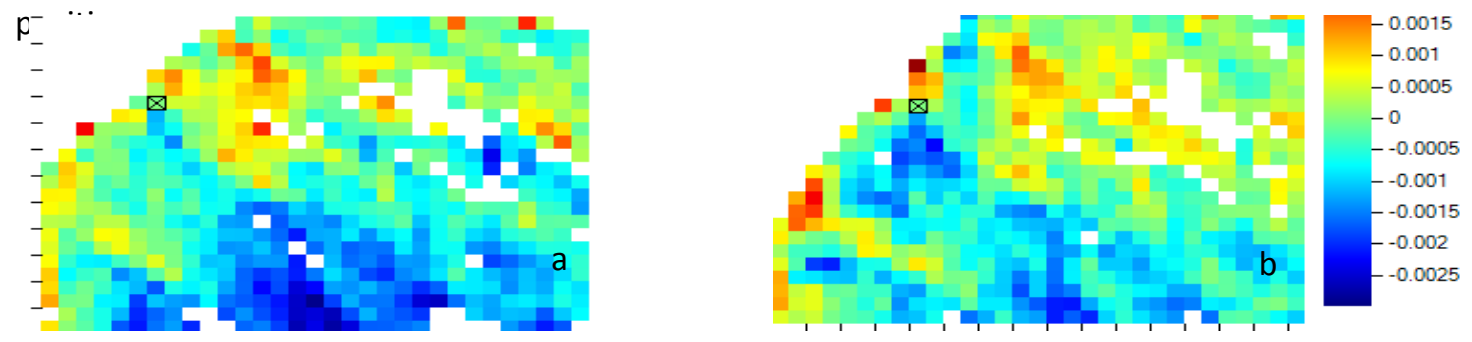

Figure 4 Sequential mapping of deformed polycrystalline nickel base alloy. (map step size $0.5 \mu \mathrm{m}$. Ref position marked with crosses)

References

[1] A.J.Wilkinson, G. Meaden, and D.J. Dingley, Ultramicroscopy, 106 (2006). p. 307.

[2] European Patent Application No. 12717453.0 